\title{
J.L. Lanciego · J.M. Giménez-Amaya \\ Notes on the combined use of V-VIP and DAB peroxidase substrates for the detection of colocalising antigens
}

Accepted: 20 January 1999

\begin{abstract}
The purpose of the present report was to investigate to what extent the new peroxidase substrate Vector VIP (V-VIP) can be used in combination with DAB chromogen for the unequivocal and permanent detection of colocalising antigens within a single neurone, according to a two-colour paradigm. With this aim, retrograde tract-tracing with cholera toxin B subunit (CTB) or fluoro-gold (FG) was performed to disclose individual, identified subpopulations of neurones in the primate substantia nigra projecting to the caudate nucleus or to the putamen, respectively. Each tracer was detected by means of a PAP procedure and finally stained brown using DAB as a chromogen. Subsequently, both series of sections were processed for the immunocytochemical detection of tyrosine hydroxylase (TH). THimmunoreactive neurones were stained purple with the peroxidase substrate V-VIP. As a result of the present procedure, several cell bodies of projection neurones, stained brown, can easily be identified within the primate substantia nigra. Some of these neurones additionally displayed purple TH immunoreaction product located in the neuronal dendrites. By contrast, CTB- or FGunlabelled neurones only show the typical purple precipitate that belongs to V-VIP substrate, both in the cell body as well as in the dendrites.
\end{abstract}

\section{Introduction}

Studies focused on the simultaneous detection of more than one marker within a single cell body have occupied neuroscientists for the past few decades. Dual-, tripleor even quadruple-labelling tract-tracing experiments with different fluorescent dyes were performed in order to disclose axonal collateralisations (Van den Pol et al. 1978; Björklund and Skagerberg 1979; Kuypers et al.

J.L. Lanciego (- J.M. Giménez-Amaya

Departamento de Anatomía, Facultad de Medicina,

Universidad de Navarra, Irunlarrea s/n, E-31080 Pamplona, Spain

e-mail: jlanciego@unav.es

Tel.: +34-948-425600 ext. 6235; Fax: +34-948-425649
1980; Alheid et al. 1984; Bentivoglio and Molinari 1984; Akintunde and Buxton 1992). Dual immunofluorescence is currently the most conventionally used method towards studying the colocalisation of various markers in a single cell body or cellular processes. The relatively new introduction of confocal laser scanning microscopy (CLSM) must be seen as an important tool, probably definitive, for the study of colocalising antigens (Mossberg et al. 1990; Wilson 1990; Brelje et al. 1993; Ulfhake et al. 1994; Belichenko and Dahlström 1995; Brismar et al. 1995; Maxwell et al. 1996; Brismar and Ulfhake 1997; Wouterlood et al. 1998).

Despite the wide acceptance of fluorescence microscopy, a different strategy was often conducted for the study of colocalising antigens by using permanent and stable peroxidase substrates. Each antigen was detected with a different chromogen, therefore, obtaining two different coloured precipitates. Although there are many chromogens currently available (Trojanowski et al. 1983), only a few of them render different colours and textures at both the light and electron microscopic levels, respectively. Especially noticeable among these we found tetramethylbenzidine (TMB), 3,3' diaminobenzidine (DAB), benzidine dihydrochloride (BDHC) and Vector VIP (V-VIP). A number of combinations between these chromogens have already been addressed (Hsu and Soban 1982; Liposits et al. 1983; Van den Pol 1985; Görcs et al. 1986; Lakos and Basbaum 1986; Levey et al. 1986; Norgren and Lehman 1989; Smith and Bolam 1992; Zhou and Grofova 1995).

Although the reliability of V-VIP substrate for double- (Zhou and Grofova 1995) or even triple-labelling experiments (Lanciego et al. 1997, 1998a,b) has been demonstrated, it remains to be verified whether or not this substrate is compatible with DAB in methodologies aimed at detecting two colocalising antigens within the same structure. The primate nigrostriatal pathway has been chosen for testing purposes (Fig. 1). Nigrostriatal projection neurones are found to be dopaminergic (Fallon and Loughlin 1985, 1995; Fallon et al. 1985). Individual projection neurones of the substantia nigra were 
Fig. 1A-C Schematic representation of the experimental design carried out in the present report and the expected results. A Illustration of the organisation of the nigrostriatal pathway: two neurones project to different areas of the caudate nucleus, while another two neurones send their axons to separate territories of the putamen. B Illustration of one injection of cholera toxin B subunit (CTB) in the caudate nucleus and the subsequent labelling obtained in the substantia nigra. Only the neurone sending its axon to the area of the caudate nucleus in which the tracer was deposited was retrogradely labelled with CTB, resulting in the deposit of a brown 3,3'diaminobenzidine (DAB) precipitate in the neuronal soma.

C Next and final step, consisted of a PAP procedure aimed at disclosing the tyrosine hydrolase $(\mathrm{TH})$-containing neurones. The substantia nigra finally stained contains two different neuronal populations: single TH-labelled neurones [purple stained with Vector VIP (V-VIP) substrate] and double-labelled neurones (CTB and $\mathrm{TH}$ ), the latter showing brown $\mathrm{DAB}$ reaction product restricted to the soma and initial dendritic segments (CTB labelling) as well as purple V-VIP reaction product located in the dendrites (TH labelling)

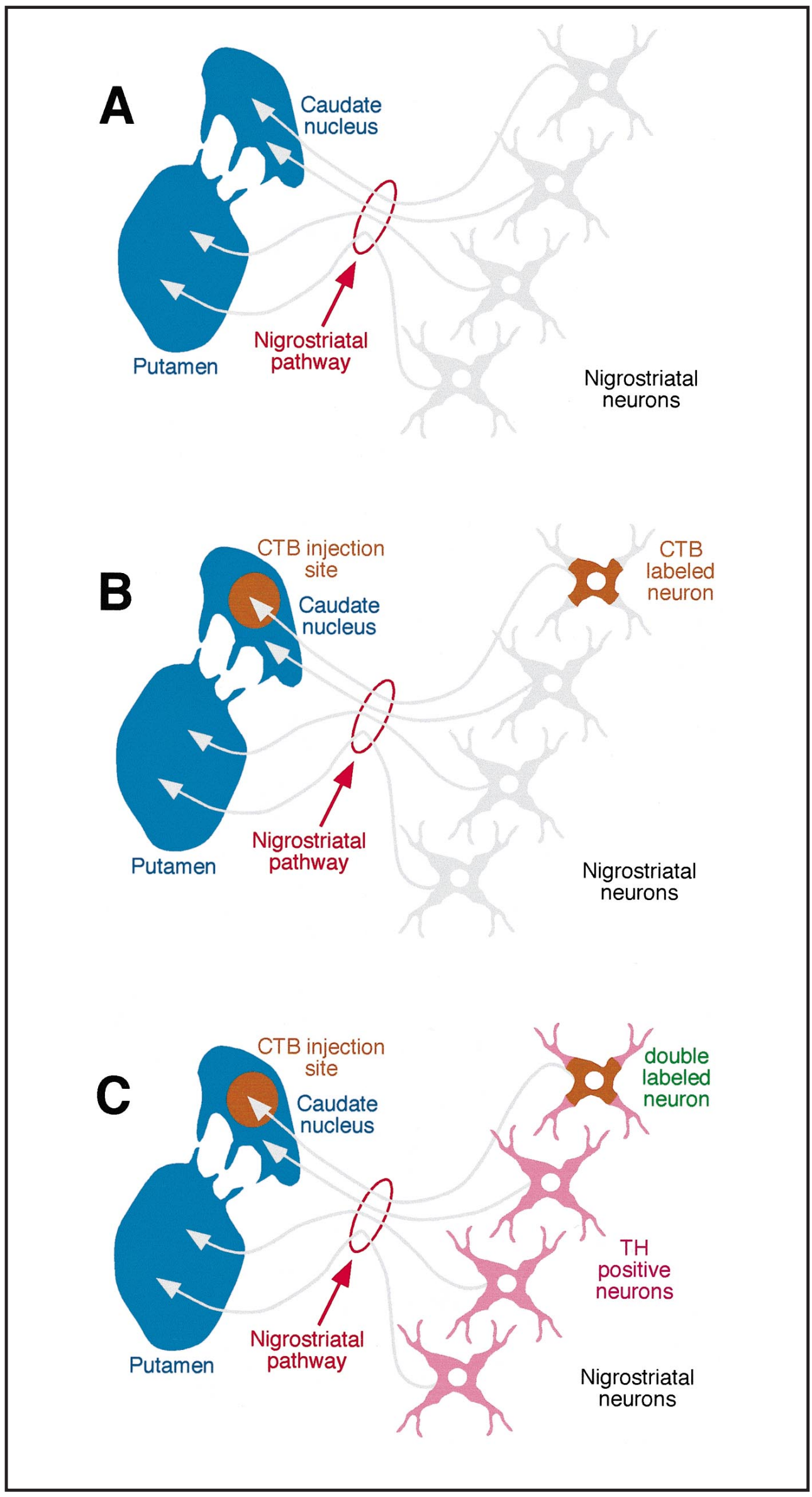


labelled after the deposit in the caudate nucleus or in the putamen of the somatopetally transported tracers, cholera toxin B subunit (CTB; Stoeckel et al. 1977; Trojanowski et al. 1981, 1982; Wan et al. 1982; Ericson and Blomqvist 1988) or Fluoro-Gold (FG; Schmued and Fallon 1986), respectively, while the dopaminergic neurones of the substantia nigra were indirectly detected via the application of a monoclonal antibody against tyrosine hydroxylase $(\mathrm{TH})$.

\section{Materials and methods}

Male Macaca fascicularis primates $(n=5)$ with a body weight ranging from 2500 to $3800 \mathrm{~g}$ were used in this study. Animals were handled at all times according to the European Council Directive 86/609/EEC as well as to the Society for Neuroscience Policy on the Use of Animals in Neuroscience Research.

Surgery, perfusion, fixation and sectioning

Anaesthesia was induced by an intramuscular injection of a mixture (1:1) containing Ketolar (ketamine, $10 \mathrm{mg} / \mathrm{ml}$ ) and Dormicum (midazolam, $5 \mathrm{mg} / \mathrm{ml}$ ). Local anaesthesia was obtained with $\mathrm{Xi}$ lonibsa (lidocaine, $10 \%$ ). Immediately before recovery, analgesia was achieved with an intramuscular injection of Finadyne (flunixin meglumine, $5 \mathrm{mg} / \mathrm{kg}$ ). This injection was repeated 24 and $48 \mathrm{~h}$ postsurgery. In order to prevent infections, one daily intramuscular injection of Ampicilina (ampicillin, $1 \mathrm{mg}$ ) was administered through an entire week.

The primates were placed in a stereotaxic frame and tracers were pressure injected with a Hamilton syringe $(0.2 \mu \mathrm{l}$ each $)$ in a single surgical session. FG (Fluorochrome, Englewood, Colo., USA) was injected in the putamen $(n=4)$ or in the caudate nucleus $(n=1)$ as a $2 \%$ solution in $0.1 \mathrm{M}$ cacodylate buffer, $\mathrm{pH}$ 7.3. CTB (List Biological Laboratories, Campbell, Calif., USA) was delivered in the ipsilateral caudate nucleus $(n=4)$ or in the putamen $(n=1)$ as a $2 \%$ solution in $0.1 \mathrm{M}$ phosphate buffer $(\mathrm{PB}), \mathrm{pH}$ 6. Finally, biotinylated dextran amine (BDA; Molecular Probes Europe, Leiden, The Netherlands) was injected in the medial part of the ipsilateral globus pallidus as a $10 \%$ solution in $0.01 \mathrm{M} \mathrm{PB}, \mathrm{pH}$ 7.25. Neuroanatomical tracing with BDA in primates belongs to our standard surgical protocols in primates, although this tracer is not related with the present report. Two initial ventriculographies with Rx contrast (Omnigraph 300; Juste SAQF, Madrid, Spain) were performed as controls, trying to find the real brain size as well as the most appropriate, horizontal position of the head. Furthermore, one additional ventriculography was done once each syringe was kept in the corresponding target area, for better accuracy. Stereotaxic coordinates were taken from the atlas of Szabo and Cowan (1984).

Two weeks postsurgery, the animals were deeply anaesthetised with an overdose of $10 \%$ chloral hydrate in distilled water and perfused transcardially. The perfusates consisted of a Ringer's saline rinsing solution, followed by $3000 \mathrm{ml}$ fixative containing $4 \%$ paraformaldehyde, $0.1 \%$ glutaraldehyde and $0.2 \%$ saturated picric acid in $0.125 \mathrm{M} \mathrm{PB}, \mathrm{pH}$ 7.4. Next, perfusion was completed with $1000 \mathrm{ml}$ of a cryoprotective solution containing 1\% DMSO and $10 \%$ glycerine in $0.125 \mathrm{M} \mathrm{PB}, \mathrm{pH} 7.4$. Immediately afterwards, the skull was opened and the brain removed. Brain blocks $(1.5 \mathrm{~cm}$ thick) were stored for $48 \mathrm{~h}$ in a second cryoprotective solution containing $2 \%$ DMSO and $20 \%$ glycerine in $0.125 \mathrm{M} \mathrm{PB}, \mathrm{pH} 7.4$. Cryoprotective solutions were prepared according to Rosene et al. (1986). Frozen coronal sections $(40 \mu \mathrm{m}$ thick) were collected in $0.125 \mathrm{M} \mathrm{PB}, \mathrm{pH}$ 7.4. A total number of ten series were obtained. One series was processed for a triple-staining procedure combining BDA + CTB + FG (Lanciego et al. 1988a) while two series of adjacent sections were processed for BDA $+\mathrm{CTB}+\mathrm{TH}$ and BDA $+\mathrm{FG}+\mathrm{TH}$, respectively. The remaining series were kept in the deep freezer $\left(-85^{\circ} \mathrm{C}\right)$ in the second cryoprotective solution for further processing. According to our experience, no apparent loss of staining quality was noticed in sections stored for at least 2 years under these conditions.

\section{Staining procedure}

For illustrative purposes, we will focus on the staining method conducted for the combination of BDA $+\mathrm{FG}+\mathrm{TH}$. Only minor differences might be observed when trying to combine BDA + CTB $+\mathrm{TH}$. An extensive, stepwise procedure for a similar triple-staining procedure $(\mathrm{BDA}+\mathrm{CTB}+\mathrm{FG})$ can be found elsewhere (Lanciego et al. 1988a). Briefly, the BDA protocol (Veenman et al. 1992) was conducted using an ABC solution (ABC kit standard; Vector, Burlingame, Calif., USA) for $90 \mathrm{~min}$. Next, the sections were reacted for 5-10 min in nickel-enhanced DAB (DAB from Sigma, St. Louis, Mo., USA). Subsequently, sections were incubated in a cocktail solution of primary antisera, comprising 1:2000 rabbit anti-FG (Chemicon, Temecula, Calif., USA) and 1:500 mouse anti-TH (Boehringer, Mannheim, Germany), for $60 \mathrm{~h}$ at $4^{\circ} \mathrm{C}$. Sections were then incubated for $2 \mathrm{~h}$ at room temperature (RT) in another cocktail solution containing 1:50 swine anti-rabbit (Dako, Copenhagen, Denmark) and 1:50 goat anti-mouse (Dako). The next step consisted of an incubation in a PAP complex raised in rabbit (1:600 rabbit-PAP; Dako) for $90 \mathrm{~min}$ at RT. The FG protocol was completed by using a regular solution of DAB as a chromogen (20-40 min, RT). The final step of the TH protocol consisted of an incubation with a PAP complex developed in mouse (1:100 mouse-PAP; Dako; $90 \mathrm{~min}$, RT), followed by $5-10 \mathrm{~min}$ staining in a V-VIP solution (Vector). V-VIP solution was prepared by adding one drop of each vial (V-VIP substrate is presented as a kit containing four vials) to $3.5 \mathrm{ml} 0.05 \mathrm{M}$ TRIS/HCl, $\mathrm{pH}$ 7.6. Finally, sections were mounted with a $2 \%$ solution of gelatine made in $0.05 \mathrm{M}$ TRIS/HCl pH 7.6, dried, dehydrated in toluene (V-VIP is very sensitive to ethanol) and coverslipped with Entellan (Merck, Darmstadt, Germany). Extensive washing with 0.05 M TBS-Tx, $\mathrm{pH} 8$, was carried out throughout the procedure. Several rinsing steps with $0.05 \mathrm{M}$ TRIS/HCl, $\mathrm{pH}$ 7.6, were performed prior and after the reactions in the different peroxidase substrates. Throughout these reactions, the progress of each reaction was inspected in a microscope at 5-min intervals. All antisera were diluted in $0.05 \mathrm{M}$ TBS-Tx, pH 8. The cocktail solution of primary antisera also contained 2\% BSA (Merck) to minimise background staining.

\section{Results}

The distribution of the different labelled neuronal populations within the primate substantia nigra was in agreement with previous studies (Fallon et al. 1978; Fallon and Loughlin 1985, 1995) and will not be discussed here in full detail. Briefly, the neurones of the substantia nigra pars compacta (SNC) projecting to the caudate nucleus or to the putamen were found to be mainly distributed in clusters of cells. Although the labelled neurones located within a single cluster showed a marked preference for one of the two types of retrograde tracers, there are other areas of the SNC in which both populations of neurones can be identified. No noticeable differences in the distribution and extent of the neuronal labelling were found when comparing the series in which both tracers were simultaneously stained (multiple tracing with BDA, CTB and FG) with the adjacent series in which each tracer is separately detected (dual tracing with BDA and CTB or FG plus the immunocytochemical detection of TH). The application of the excellent antibody against $\mathrm{TH}$ always 

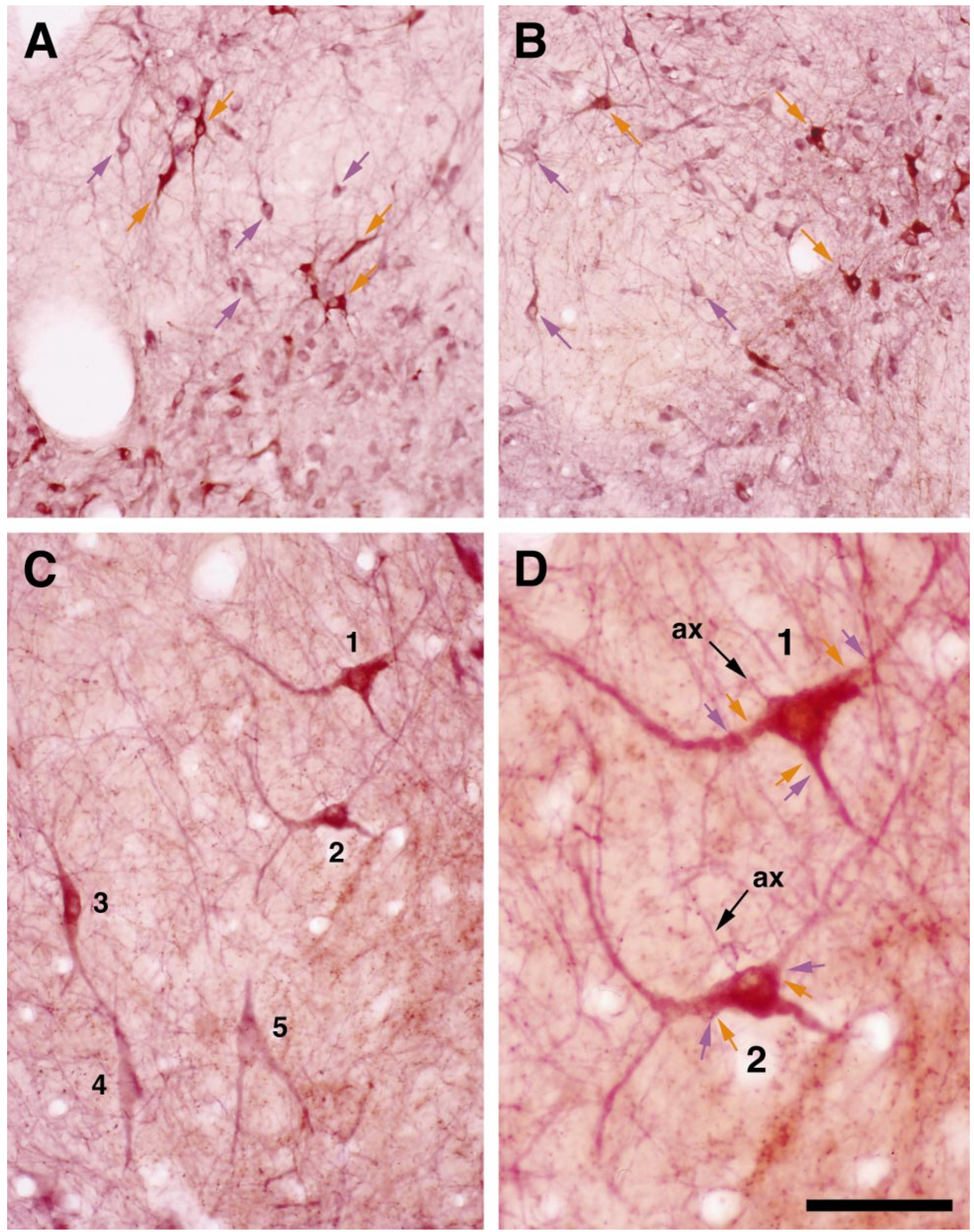

resulted in massive, Golgi-like neuronal labelling throughout the primate substantia nigra, and mostly it was possible to follow their dendritic arborisations over long distances (Fig. 2). The V-VIP reaction product is characterised by a diffuse purple precipitate.
Several attempts were made in testing the most appropriate sequence for the application of the chromogens in order to reduce non-specific background staining as well as colour mixing phenomena. The best results were achieved when DAB peroxidase substrate was used first, 
followed by V-VIP chromogen as the last one to be used. This sequence allowed us to use the strongest chromogen (DAB) to disclose an small subpopulation of neurones (neurones showing the transported marker), while the weakest chromogen (V-VIP) is devoted to the detection of TH-immunoreactive cells, a much larger population than the former one. When V-VIP chromogen is used first for the detection of the transported marker, the subsequent $\mathrm{TH}$ stain with $\mathrm{DAB}$ peroxidase substrate often masked the previously obtained results.

Distinct differences in the texture of the final DAB reaction product were found when this chromogen was used for the detection of CTB- or FG-labelled neurones, the latter showing a typical granular precipitate while the CTB-labelled neurones were characterised by a more diffuse brown reaction product. More importantly, some marked differences in the distribution of the DAB precipitate within the neuronal soma and processes were also observed. As previously reported elsewhere (Schmued and Fallon 1986), somatopetally transported FG often resulted in Golgi-like neuronal labelling, while transported CTB is more restricted to the neuronal soma as well as to the initial segments of the principal dendrites (Fig. 2A,B).

The reliability of the combined use of DAB and VVIP peroxidase substrates for the staining of colocalising antigens varies as a function of the antigens themselves. In our experience, the best results were obtained when trying to detect the transported CTB colocalising with TH-immunoreactive cells (Fig. 2C,D). As pointed out before, the transported CTB, stained brown with $\mathrm{DAB}$, is often restricted to the neuronal soma and the initial portions of the main dendrites, without passing onto the distal dendrites. These distal processes were then occupied by the purple reaction product obtained from the TH protocol. By contrast, the Golgi-like appearance of the FG-

Fig. 2A-D The combined use of DAB and V-VIP peroxidase substrate for the detection of colocalising antigens. A Microphotograph taken from one section of the substantia nigra processed for dual labelling with fluoro-gold (FG) and TH. Retrogradely labelled neurones with FG (brown arrows) were characterised by a diffuse brown reaction product (DAB). The $\mathrm{DAB}$ precipitate was localised in the neuronal soma as well as in the dendrites, to some distance. By contrast, TH-immunoreactive neurones were labelled purple with V-VIP. B Microphotograph taken from one section of the substantia nigra processed for dual labelling with CTB and TH. Brown arrows indicate CTB-labelled neurones and purple arrows shows TH-immunoreactive cells. By comparing this picture with the former one, please note that the $\mathrm{DAB}$ reaction product that belongs to the retrogradely transported CTB is mainly restricted to the neuronal soma, while the DAB precipitate corresponding to the somatopetally transported FG goes onto the dendrites to a much higher extent. C Comparison between single TH-immunoreactive-labelled cells (neurones numbered 4 and 5) and double CTB + TH-labelled cells (neurones numbered 1, 2 and 3). D High magnification picture showing a pair of double-labelled cells with CTB and TH. Transported CTB is labelled in brown with DAB and restricted to the neuronal soma, while the purple V-VIP reaction product corresponding to the TH immunoreaction is located in the dendrites, which can be followed to some distance. ax Initial segments of the axons, purple-labelled. Several pairs of arrows (purple and brown) illustrate the border between both coloured reaction products. Scale bar $50 \mu \mathrm{m}$ for $\mathbf{A}, \mathbf{B} ; 25 \mu \mathrm{m}$ for $\mathbf{C} ; 12.5 \mu \mathrm{m}$ for $\mathbf{D}$ labelled neurones often hampers the subsequent staining for $\mathrm{TH}$, because the neuronal soma and their entire processes were labelled brown with DAB.

\section{Discussion}

\section{Dual labelling with DAB and V-VIP}

The final related precipitates (brown vs purple) were colours distinct enough to provide an unequivocal detection of the labelled antigens. Overall, this procedure can be applied for the detection of any pair of antigens, although there are several limitations requiring further discussion. Firstly, the order in which the chromogens have to be applied played an important role in the final staining quality. The weakest chromogen, V-VIP, should be the last one to be used, in order to maintain its brilliant purple colour, while the strong DAB substrate was always employed for the first reaction. Secondly, special attention should be given to the subcellular localisation of each of the antigens within the neurone. In other words, the best results will be accomplished when both antigens were localised, for example, in the neuronal nucleus and in the cytoplasm, respectively. It has been demonstrated that V-VIP substrate has a granular texture under the electron microscope and its successful combination with $\mathrm{DAB}$ in double pre-embedding procedures has already been described (Zhou and Grofova 1995). Nevertheless, further research is needed to investigate the final texture resulting from the addition of the granular V-VIP reaction product to the flocculent DAB precipitate when staining two antigens within the same neuronal profile.

\section{Alternative procedures for permanent dual labelling}

There are currently available few chromogens that may be used for paired antigen localisation at the light and electron microscopy levels. Especially noticeable among these is the combination of TMB and DAB (Norgren and Lehman 1989), as well as methods combining DAB and BDHC (Lakos and Basbaum 1986; Levey et al. 1986). These methodologies have proven to be effective, although some differences in sensitivity among TMB and BDHC chromogens has been reported (Levey et al. 1985). Furthermore, problems related to BDHC background staining and stability of the final precipitate have also been described (Levey et al. 1985). A different point of view was provided by Tramu et al. (1978). These authors reported an efficient method for antibody elution by removing the antisera retained by tissue antigen after immunohistochemical staining. Antisera removal was achieved by using $\mathrm{KMnO}_{4}-\mathrm{H}_{2} \mathrm{SO}_{4}$ as an oxidising agent, followed by reduction with $\mathrm{Na}_{2} \mathrm{~S}_{2} \mathrm{O}_{5}$. Despite the good colour segregation in light microscopy obtained with combinations of DAB and TMB or BDHC, at the electron microscopic level these chromogens are characterised by a crystalline deposit, sometimes obscuring fine 
morphological detail. Although the colour segregation obtained with the use of DAB and V-VIP substrates for light microscopy is good enough, there currently is little known on the simultaneous application of DAB and VVIP chromogens for the study of colocalising antigens at the ultrastructural level. The granular electron-dense precipitate that belongs to V-VIP substrate (Zhou and Grofova 1995) allows the unequivocal detection of the labelled structures and easy identification of their subcellular compounds without compromising fine ultrastructural detail, and these characteristics seems to be very attractive for further testing.

\section{Methodologies based on fluorescence microscopy}

These methods have been formerly used to disclose the chemical nature of projection neurones, by combining fluorescent histochemistry with retrograde fluorescent tracers (Björklund and Skagerberg 1979), as well as to study divergent axonal collateralisation (Kuypers et al. 1980). Multiple retrograde tracing with fluorescent dyes faces several drawbacks, such as fading phenomena, tracer diffusion, false positives and competition among the involved tracers. Although a few of these drawbacks can be avoided (Alheid et al. 1984; Bentivoglio and Molinari 1984; Balercia et al. 1992), the arrival of new technologies quickly took the lead in multiple fluorescence studies.

The most widely used method towards answering the question whether or not colocalisation exists in neurones of two different neuronal antigens is double-immunofluorescence microscopy. This method relies on the use of dye-coupled antisera with different absorption/emission spectra (Chang and Kuo 1989; Wessendorf 1990; Campbell et al. 1991; Lowy 1995; GonzaloRuiz et al. 1996; Liang et al. 1996; Scalettar et al. 1996; Saffiedine et al. 1997; Hajos et al. 1998; Wouterlood et al. 1998). Nevertheless, direct visualisation under epifluorescence may resulted in important pitfalls, such as false positives, spherical aberration, overlapping of absorption/emission spectra among fluoroprobes and difficulties in controlling the depth of focus (Lowy 1995; Scalettar et al. 1996; Wouterlood et al. 1998). In this regard, the consequent use of the CLSM (Wilson 1990; Brelje et al. 1993; Ulfhake et al. 1994) has been demonstrated to be an excellent choice to circumvent these problems. Although some specific precautions have to be exerted during the examination of nervous tissue under the CLSM (Ulfhake et al. 1994; Brismar et al. 1995; Wouterlood et al. 1998) this equipment probably is the most accurate strategy currently available for the study of colocalising antigens.

Acknowledgements Expert technical assistance was provided by Ms A. Moreno. Supported by F.I.S. No. 96/0488, Fundación "Marcelino Botín" and University of Navarra

\section{References}

Akintunde A, Buxton DF (1992) Quadruple labeling of brain-stem neurons: a multiple retrograde fluorescent tracer study of axonal collateralisation. J Neurosci Methods 45:15-22

Alheid GF, Carlsen J, De Olmos J, Heimer L (1984) Quantitative determination of collateral anterior olfactory nucleus projections using a fluorescent tracer with an algebraic solution to the problem of double retrograde labeling. Brain Res 292:17-22

Balercia G, Chen S, Bentivoglio M (1992) Electron microscopic analysis of fluorescent neuronal labeling after photoconversion. J Neurosci Methods 45:87-98

Belichenko PV, Dahlström A (1995) Mapping of human brain in normal and pathological situations: the single cell and the fiber level, employing Lucifer Yellow microinjection, carbocyanine dye tracing, immunofluorescence, and 3D confocal laser scanning microscopy reconstruction. Neurosci Prot 95-050-03-01

Bentivoglio M, Molinari M (1984) Fluorescent retrograde triple labeling of brainstem reticular neurons. Neurosci Lett 46:121-126

Björklund A, Skagerberg G (1979) Simultaneous use of fluorescent tracers and fluorescence histochemistry for convenient and precise mapping of monoaminergic projections and collateral arrangements in the CNS. J Neurosci Methods 1:261-277

Brelje TC, Wessendorf RL, Sorenson RL (1993) Multicolor laser scanning confocal immunofluorescence microscopy: practical application and limitations. In: Matsumoto B (ed) Methods in cell biology. Cell biological applications in confocal microscopy, vol 38. Academic Press, Sydney, pp 98-181

Brismar H, Ulfhake B (1997) Fluorescence lifetime measurements in confocal microscopy of neurons labeled with multiple fluoroprobes. Nature Biotechnol 15:373-377

Brismar H, Trepte O, Ulfhake B (1995) Spectra and fluorescence lifetimes of lissamine rhodamine, tetramethylrhodamine isothiocyanate, texas red, and cyanine 3.18 fluoroprobes: influences of some environmental factors recorded with a confocal laser scanning microscope. J Histochem Cytochem 47:699-707

Campbell KJ, Takada M, Hattori T (1991) Co-localization of tyrosine hydroxylase and glutamate decarboxylase in a subpopulation of single nigrotectal projection neurons. Brain Res 558: 239-244

Chang HT, Kuo H (1989) Adrenergic innervation of the substantia innominata: co-localization of phenylethanolamine $N$-methyltransferase and tyrosine hydroxylase immunoreactivities within the same axons. Brain Res 503:350-353

Ericson H, Blomqvist A (1988) Tracing of neuronal connections with cholera toxin subunit B: light and electron microscopic immunohistochemistry using monoclonal antibodies. J Neurosci Methods 24:225-235

Fallon JH, Loughlin SE (1985) The substantia nigra. In: Paxinos G, Watson J (eds) The rat central nervous system: a handbook for neuroscientists. Academic Press, Sydney, pp 353-374

Fallon JH, Loughlin SE (1995) Substantia nigra. In: Painos G (ed) The rat nervous system, 2nd edn. Academic Press, Sydney, pp 215-237

Fallon JH, Riley J, Moore RY (1978) Substantia nigra dopamine neurons: separate populations project to neostriatum and allocortex. Neurosci Lett 7:157-162

Gonzalo-Ruiz A, Sanz JM, Lieberman AR (1996) Immunohistochemical studies of localization and co-localization of glutamate, aspartate and GABA in the anterior thalamic nuclei, retrosplenial granular cortex, thalamic reticular nucleus and mammilary nuclei of the rat. J Chem Neuroanat 12:77-84

Görcs T, Leranth C, MacLusky NJ (1986) The use of gold substituted silver-intensified diaminobenzidine (DAB) and non-intensified DAB for simultaneous electron microscopic labeling of tyrosine hydroxylase and glutamic acid decarboxylase immunoreactivity in the rat medial preoptic area. J Histochem Cytochem 34:1439-1447

Hajos N, Papp,EC, Acsady L, Levey AI, Freund TF (1998) Distinct interneuron types express $\mathrm{m} 2$ muscarinic receptor immunoreactivity on their dendrites or axon terminals in the hippocampus. Neuroscience 82:355-376 
Hsu HS, Soban E (1982) Color modification of diaminobenzidine (DAB) precipitation by metallic ions and its application for double immunohistochemistry. J Histochem Cytochem 30: 1079-1082

Kuypers HGJM, Bentivoglio M, Catsman-Berrevoets CE, Bharos AT (1980) Double retrograde neuronal labeling through divergent axon collaterals, using two fluorescent tracers with the same excitation wavelength which label different features of the cell. Exp Brain Res 40:383-392

Lakos S, Basbaum AI (1986) Benzidine dihydrochloride as a chromogen for single- and double-label and electron microscopic immunocytochemical studies. J Histochem Cytochem 34: $1047-1056$

Lanciego JL, Goede PH, Witter MP, Wouterlood FG (1997) Use of peroxidase substrate Vector VIP® for multiple staining in light microscopy. J Neurosci Methods 74:1-7

Lanciego JL, Luquin MR, Guillén J, Giménez-Amaya JM (1998a) Multiple neuroanatomical tracing in primates. Brain Res Prot $2: 323-332$

Lanciego JL, Wouterlood FG, Erro E, Giménez-Amaya JM (1998b) Multiple axonal tracing: simultaneous detection of three tracers in the same section. Histochem Cell Biol 110:509-515

Levey AI, Bolam JP, Rye DB, Hallanger AE, Demuth RM, Mesulam MM, Wainer BH (1986) A light and electron microscopic procedure for sequential double antigen localization using diaminobenzidine and benzidine dihydrochloride. J Histochem Cytochem 34:1449-1457

Liang CL, Sinton CM, German DC (1996) Midbrain dopaminergic neurons in the mouse: co-localization with calbindin-D28 K and calretinin. Neuroscience 75:523-533

Liposits Z, Görcs T, Torök A, Domány S, Sétaló G (1983) Simultaneous localization of two different tissue antigens based on the silver intensified PAP-DAB and the traditional PAP-DAB methods. Acta Morphol Acad Sci Hung 31:356-369

Lowy RJ (1995) Evaluation of triple-band filters for quantitative epifluorescence microscopy. J Microsc 178:240-250

Maxwell L, Maxwell DJ, Neilson M, Kerr R (1996) A confocal microscopic survey of serotoninergic axons in the lumbar spinal cord of the rat: co-localization with glutamate decarboxylase and neuropeptides. Neuroscience 75:471-480

Mossberg K, Arvidsson U, Ulfhake B (1990) Computerized quantification of immunofluorescence-labeled axon terminals and analysis of co-localization of neurochemicals in axon terminals with a confocal scanning laser microscope. J Histochem Cytochem 38:179-190

Norgren RB, Lehman MN (1989) A double-label pre-embedding immunoperoxidase technique for electron microscopy using diaminobenzidine and tetramethylbenzidine as markers. J Histochem Cytochem 37:1283-1289

Rosene DL, Roy NJ, Davis BJ (1986) A cryoprotection method that facilitates cutting frozen sections of whole monkey brain for histological and histochemical processing without freezing artifact. J Histochem Cytochem 34:1301-1316

Saffiedine S, Prior AM, Eybalin M (1997) Choline acetyltransferase, glutamate decarboxylase, tyrosine hydroxylase, calcitonin gene-related peptide and opioid peptides coexist in lateral efferent neurons of rat and guinea pig. Eur J Neurosci 9:356-367

Scalettar BA, Swedlow JR, Sedat JW, Agard DA (1996) Dispersion, aberration and deconvolution in multi-wavelength fluorescent images. J Microsc 182:50-60

Schmued LC, Fallon JH (1986) Fluoro-gold: a new fluorescent retrograde axonal tracer with numerous unique properties. Brain Res 377:147-154
Smith Y, Bolam JP (1992) Combined approaches to experimental neuroanatomy: combined tracing and immunocytochemical techniques for the study of neuronal microcircuits. In: Bolam JP (ed) Experimental neuroanatomy, a practical approach. Oxford University Press, Oxford, pp 239-266

Stoeckel K, Schwab ME, Thoenen H (1977) Role of gangliosides in the uptake and retrograde axonal transport of cholera and tetanus toxin as compared to nerve growth factor and wheat germ agglutinin. Brain Res 132:273-285

Szabo J, Cowan WM (1984) A stereotaxic atlas of the brain of the cynomolgus monkey (Macaca fascicularis). J Comp Neurol 222:265-300

Tramu G, Pillez A, Leonardelli J (1978) An efficient method of antibody elution for the successive or simultaneous localization of two antigens by immunocytochemistry. J Histochem Cytochem 26:322-324

Trojanowski JQ, Gonatas JO, Gonatas NK (1981) Conjugates of horseradish peroxidase (HRP) with cholera toxin and wheat germ agglutinin are superior to free HRP as orthograde transported markers. Brain Res 223:381-385

Trojanowski JQ, Gonatas JO, Gonatas NK (1982) Horseradish peroxidase (HRP) conjugates of cholera toxin and lectins are more sensitive retrogradely transported markers than free HRP. Brain Res 231:33-50

Trojanowski JQ, Obrocka MA, Lee VMY (1983) A comparison of eight different chromogen protocols for the demonstration of immunoreactive neurofilaments or glial filaments in rat cerebellum using the peroxidase-antiperoxidase method and monoclonal antibodies. J Histochem Cytochem 31:1217-1223

Ulfhake B, Carlsson K, Mossberg K, Wallén P (1994) Preparation, staining and examination of nervous tissue in the confocal scanning laser microscope. Neurosci Prot 94-050-01-01-28

Van den Pol AN (1985) Silver-intensified gold and peroxidase as dual ultrastructural immunolabels for pre- and postsynaptic neurotransmitters. Science 228:332-335

Van der Kooy D, Kuypers HGJM, Catsman-Berrevoets C (1978) Single mammilary body cells with divergent axon collaterals. Demonstration by a simple, fluorescent retrograde double labeling technique in the rat. Brain Res. 158:189-196

Veenman CL, Reiner A, Honig MG (1992) Biotinylated dextran amine as an anterograde tracer for single- and double-label studies. J Neurosci Methods 41:239-254

Wan SXC, Trojanowski JQ, Gonatas JO (1982) Cholera toxin and wheat germ agglutinin conjugates as neuroanatomical probes: their uptake and clearance, transganglionic and retrograde transport and sensitivity. Brain Res 243:215-224

Wessendorf MW (1990) Characterization and use of multi-color fluorescence microscopic techniques. In: Bjorklund A, Wouterlood FG, Van den Pol AN (eds) Handbook of chemical neuroanatomy, vol 8. Elsevier, Amsterdam, pp 1-41

Wilson T (1990) Confocal microscopy. Academic Press, Sydney

Wouterlood FG, Van Denderen JCM, Blijleven N, Van Minnen J, Härting W (1998) Two-laser dual-immunofluorescence confocal laser scanning microscopy using $\mathrm{Cy} 2-$ and $\mathrm{Cy} 5$-conjugated secondary antibodies: unequivocal detection of co-localization of neuronal markers. Brain Res Prot 2:149-159

Wurden S, Homberg U (1993) A simple method for immunofluorescent double staining with primary antisera from the same species. J Histochem Cytochem 41:627-630

Zhou M, Grofova I (1995) The use of peroxidase substrate Vector ${ }^{\circledR}$ VIP in electron microscopic single and double antigen localization. J Neurosci Methods 62:149-158 